\title{
Energy Proficient Reliable Rim Routing Technique for Wireless Heterogeneous Sensor Networks Lifespan Fortification
}

\author{
S. G. Susila ${ }^{1}$, J. Arputhavijayaselvi² \\ ${ }^{1}$ VF/ECE, Anna University Chennai BIT Campus, Tiruchirappalli, India \\ ${ }^{2}$ Dean R and D, Professor/ECE, Kings College of Engineering, Pudukottai, India \\ Email: "rsksgsnilajillu@gmail.com
}

Received 1 April 2016; accepted 25 April 2016; published 20 June 2016

Copyright (C) 2016 by authors and Scientific Research Publishing Inc.

This work is licensed under the Creative Commons Attribution International License (CC BY). http://creativecommons.org/licenses/by/4.0/

c) (i) Open Access

\begin{abstract}
Sensor nodes are mainly shielded in the field with limited power supply. In Wireless Sensor Networks, there must be a requirement of an efficient power management, because sensor nodes are deployed in unman attended area with non-rechargeable batteries. Power management can be done by different methods of routing protocols. The proposed Reliable Rim Routing (3R) technique is based on hybrid routing protocol for homogeneous and heterogeneous system for WSNs to ameliorate the performance of the overall system. In $3 R$, total node deployment area can be multipart in terms of rim and in each rim, and some of the sensor nodes transmit their sensed data directly to base station, and meanwhile remaining sensor nodes send the data through clustering technique to base station like SEP. Proposed 3R technique implementation proves its enhanced WSNs lifetime of $70 \%$ energy consumption and $40 \%$ throughput compared with existing protocols. Simulation and evaluation results outperformed in terms of energy consumption with increased throughput and network lifetime.
\end{abstract}

\section{Keywords}

Cluster, Cluster Head, Heterogeneous, Rim Layer Node Deployment Technique, Wireless Sensor Network

\section{Introduction}

In topical times, many pursuing researchers have shown great concentration in WSNs due to their extensive

\footnotetext{
${ }^{*}$ Corresponding author.
}

How to cite this paper: Susila, S.G. and Arputhavijayaselvi, J. (2016) Energy Proficient Reliable Rim Routing Technique for Wireless Heterogeneous Sensor Networks Lifespan Fortification. Circuits and Systems, 7, 1751-1759. 
range of the appliance in the field of military surveillance, fire detection, habitat monitoring, industry, health monitoring and many more. WSNs are serene of mammoth number of randomly deployed sensor nodes in the field. Sensor nodes sense the occurrence and propel the data to base station via single or multi-hop communication. Since most of the energy is consumed during data transfer communication, presently dissimilar clustering algorithms are projected for WSNs to use energy of nodes resourcefully. Clustering concept of implementation is utilized in WSNs to diminish energy consumption [1]. Maneuver of clustering algorithm is executed in terms of rounds in simulation. Sensor nodes are prearranged in self-governing sets or clusters. At least one cluster head is selected for each cluster in each simulation round. The sensed data are not directly sent to the base station but via respective cluster heads. Cluster head collects data of sensor nodes that belong to that cluster. Clustering algorithms apply data aggregation procedures which reduce the collected data at cluster head in the form of significant information. Cluster heads then send the aggregated data to base station. This cluster head selection methods are highly optimum for long distanced nodes which are deployed far away from base station, but closely deployed nodes are lost some additional energy to form a cluster and cluster head selection process. To avoid this hampers, it is planned to communicate the data directly to base station for energy consumption. Furthermore, the implementation of the RIM layer concept divides the total node deployment area in terms of distance from the base station.

The rest of the paper is organized as follows. The detailed surveys, concept of heterogeneity and algorithm statement which are closely related to the research work are mentioned in Section 2. Section 3 depicted the information of proposed Reliable Rim Routing (3R) implementation of its two models. Analysis of simulation results is focused in Section 4. The obtained results and its conclusions are mentioned in Section 5.

\section{Related Work}

LEACH [2] [3] is a hierarchical clustering algorithm for judicious usage of energy in the network. LEACH uses randomized rotation of the local cluster head. LEACH executes well in homogeneous and heterogeneous environment. In LEACH each sensor node has same probability to become a cluster head. However, LEACH is not well suited for heterogeneous environment due to certain reasons.

SEP [4] is a two level heterogeneous protocol introducing two types of nodes, normal nodes and advance nodes. Advance nodes have more energy than normal nodes. In SEP both nodes (normal and advance nodes) have weighted probability to become cluster head. Advance nodes have more chances to become cluster head than normal nodes. SEP does not guarantee efficient deployment of nodes. Enhanced Stable Election Protocol (E-SEP) was proposed for three level hierarchies. E-SEP introduced an intermediate node whose energy reclines between normal node and advance node. Nodes elect themselves as cluster head on the basis of their energy level comparison. The drawback of E-SEP is same as in SEP.

Distributed Energy-Efficient Clustering Protocol (DEEC) illustrates multilevel heterogeneity [5]. In DEEC, the cluster head creation is based on residual energy of node and average energy of the network and high energy node has more likelihood to become cluster head than low energy node. It contains the algorithm for set up phase and steady state phase. In setup phase, $\mathrm{CH}$ send the advertisement packet to give a message to neighbors he become the $\mathrm{CH}$. In steady state phase, there are two processes done such as schedule creation and data transmission.

In Z-SEP [6] and M-SEP [7], there are two types of nodes as normal nodes and advanced nodes. The whole area is divided into three zones as zone 0 , zone 1 and zone 2 . Zone 0 is the middle zone whereas Zone 1 and Zone 2 are the upper and lower zones respectively. In zone 0 normal nodes are deployed and they converse with the base station directly. Zone 1 and zone 2 advanced nodes are deployed and they form clusters. Then the cluster head pass the data to base station. For example, if the field consists of 100 sensor nodes, Zone 0 has 80 nodes for direct communication to BS which is located at center. Remaining 20 nodes are equally divided into Zone 1 and Zone 2 with different energy inputs. In this method of transmission, more simulation rounds in terms of energy consumptions are increased when compare to SEP procedure transmission.

\subsection{Impact of Heterogeneity Structure in Wireless Sensor Networks}

Insertion of a small number of heterogeneous [8] [9] sensor nodes in the sensor network can carry following profit. Latency decrease in data communication because of the computational heterogeneity can decrease the processing latency in immediate nodes and connection heterogeneity can reduce the waiting time in the trans- 
mitting line. Smaller amount of hops between sensor nodes to sink node also have less amount of latency.

Extending network lifespan: The average energy saving for sending a packet from the normal nodes to the sink in heterogeneous sensor networks will be much less than the energy consumed by the homogeneous sensor networks.

Ameliorate reliability of data transmission is familiar that sensor network associations have an inclination to have small reliability. Each step significantly lowers the end-to-end delivery rate. In the usage of heterogeneous sensor nodes, fewer hop between normal sensor nodes and sink. Consequently the heterogeneous sensor network can acquire much higher end-to-end delivery rate than the homogeneous sensor network. The other system improvement by utilizing heterogeneous system in WSNs yield power consumption [10], topology [11], improving reliability of data transmission [12] network lifetime, number of cluster heads per rounds, energy efficiency [13] and throughput.

Network lifetime [14] [15] (stability period): It is the time interval from the start of operation (of the sensor network) until the death of the first alive node to last dead node.

Number of cluster heads per round: Instantaneous measure reflects the number of nodes which would send directly to the base station, information aggregated from their cluster members.

Energy Efficiency [16]-[19]: The innovative ways for smart energy saving with new schemes of routing algorithms is established. Energy consumption is differs from methods of routing protocols and algorithms.

Throughput: This includes the total rate of data sent over the network, the rate of data sent from cluster heads to the base station as well as the rate of data sent from the nodes to their cluster heads.

\subsection{Details of Algorithm Implementation in the Reliable Rim Routing Technique}

This paper emphasis the incorporated algorithm description as follows. The process of formation of clusters and cluster head selection system are followed by the given procedure.

// Initial phase:

In initial phase of each round a population and fitness function is be used to select cluster head and cluster member. In first round of algorithm choose $\mathrm{K}$ initial cluster heads $\mathrm{z} 1, \mathrm{z} 2 \ldots \ldots, \mathrm{zK}$ from the $\mathrm{n}$ nodes $\{\mathrm{x} 1$, $\mathrm{x} 2, \ldots \ldots, \mathrm{xn}\}$. Then cluster member will be selected for each cluster head.

- Step1: Initially all nodes have same probability to become a cluster head.

- Step2: $\mathrm{F}(\mathrm{t})$ is the fitness function used to select the cluster head and defined as $\mathrm{F}(\mathrm{t})=(\operatorname{Er} * \mathrm{~T}(\mathrm{n}) * \mathrm{~K}$ mean), where $\mathrm{Er}=$ Residual energy of the node, $\mathrm{T}(\mathrm{n})=$ probability of node to become cluster head as given by LEACH, $\mathrm{K}$ mean $=$ Degree of coverage. $\mathrm{In}$ this equation the energy, $\mathrm{k}$ mean and probability have a direct relationship with the output of function, so increased value of inputs will produce a larger output the optimal value obtained for a particular node will be eligible to be a cluster node.

- Step 3: After calculating this built in $\mathrm{F}(\mathrm{t})$ function. All nodes sort in descending order.

- Step 4: Selection of Cluster Head.

BS selects the cluster head and the result is sent to the network. The node has minimum difference of energy from last round and with best probability according to the stable election protocol, a node act as cluster head to network. Other nodes bind to the nearest cluster head.

- Step 5: Selected cluster head will send the information to BS.

- Step 6: Cluster Formation.

Selected cluster head send advertisement to normal nodes. Non cluster head nodes join to closest $\mathrm{CH}$ to form cluster.

- Step 7: Sensing unneeded node to sleep state.

Algorithm details:

Sn: Sensor node

Sch: Cluster head

C: Cluster formed

//Setup phase:

1. for each $\mathrm{Sn}$ to $\mathrm{n}$ do

2. $\mathrm{Ci}=\{\mathrm{s} 1, \mathrm{~s} 2, \mathrm{Sn}\}$

3. Select Sch //data transmission phase //Send data to cluster head

4. $\mathrm{Ci}(\mathrm{s} 1, \mathrm{~s} 2, \mathrm{Sn})->\mathrm{Sch} / / \mathrm{Send}$ data to next cluster head or node 


\section{Sch->next Sch or Sn}

If (Energy (Sch<Threshold Energy)) then select new Sch within C

Mathematical Module:

Graphical method and set theory is used for calculation.

Let $\mathrm{S}$ be the system where

$\mathrm{S}=\{\mathrm{n}, \mathrm{Si}, \mathrm{P}, \mathrm{CH}, \mathrm{N}\}$

n: Sensor Node

Si: Sink Node

CH: Cluster Head

$\mathrm{N}$ : Network

$\mathrm{n}=\mathrm{n} 1, \mathrm{n} 2, \ldots, \mathrm{n}$

$\mathrm{Si}=$ only one sink node

$\mathrm{P}=\mathrm{P} 1, \mathrm{P} 2, \ldots, \mathrm{Pn}$

$\mathrm{CH}=$ Cluster Head (depend on cluster formation)

$\mathrm{N}=$ Network

Activity:

$\mathrm{f}(\mathrm{Si}) \mathrm{N}$-Sink node is depends on Network

$\mathrm{f}(\mathrm{CH}) \mathrm{n}$-Cluster head is depends on Node

$\mathrm{f}(\mathrm{n}) \mathrm{N}-\mathrm{Nodes}$ are included in Network

f (Si)BS-Sink Node site use by Network

\section{Proposed Reliable Rim Routing Technique}

Proposed 3R algorithm (Reliable Rim Routing), the senor field divided by rim. There are three rims present in the sensor node deployed field. The inner rim is comprised of normal node (Energy level is 0.5 Joule, Rim distance is $25 \mathrm{~m}$ ) and it can communicate the sensed data directly to the base station. The first outer rim is deployed by advanced nodes with energy of 1 joule (Rim distance is $30 \mathrm{~m}$ ) and it communicate the sensed data directly to the base station. The second rim is occupied by super advanced nodes (Energy level is 1.5 Joules) and it forms clusters, then the Cluster head transmits the data to the BS. If the distance of the rim size increases the random number of nodes inside the rim decreases and vice versa. Node $n$ choosing a random number $m$ between 0 and 1 , if $m<T(n)$ for node $n$, the node becomes a cluster-head as in Equation (1).

$$
T s(n)=\frac{p_{s}}{1-p_{s} *\left(r \bmod \frac{1}{p_{s}}\right)} \text { if } n £ G
$$

where $T_{s}(n)$-cluster head selection threshold;

$P_{s}$ : predetermined fraction of node elected as cluster head in super advanced node;

$r$ : denotes current round;

$G$ is the set of nodes that have not been cluster-heads in the last $1 / P$ rounds.

\subsection{Reliable Rim Routing Model-1}

Figure 1 shows that 3R random node deployment, 100 nodes are randomly deployed at $100 \mathrm{~m} \times 100 \mathrm{~m}$ area; a rim is inserted at $25 \mathrm{~m}$ distance from the origin of the deployment area. Inside the rim area normal nodes are deployed which is having 0.5 Joule energy, why because nodes are near to the base station that consume less energy for direct data transmission. The outer rim has heterogeneous nodes Such as advanced node which is 1 Joule energy. Heterogeneous nodes are form the cluster and cluster head selection process is adopted by using stable election protocol and transmit the data to base station.

\subsection{Reliable Rim Routing Model-2}

Figure 2 shows that $3 \mathrm{R}$ random node deployment, 100 nodes are randomly deployed at $100 \mathrm{~m} \times 100 \mathrm{~m}$ area; double rim is inserting at $20 \mathrm{~m}$ and $30 \mathrm{~m}$ distance from the origin of the deployment area. Inside the first rim area normal nodes are deployed which is 0.5 Joule energy because, nodes are near to the base station that con- 


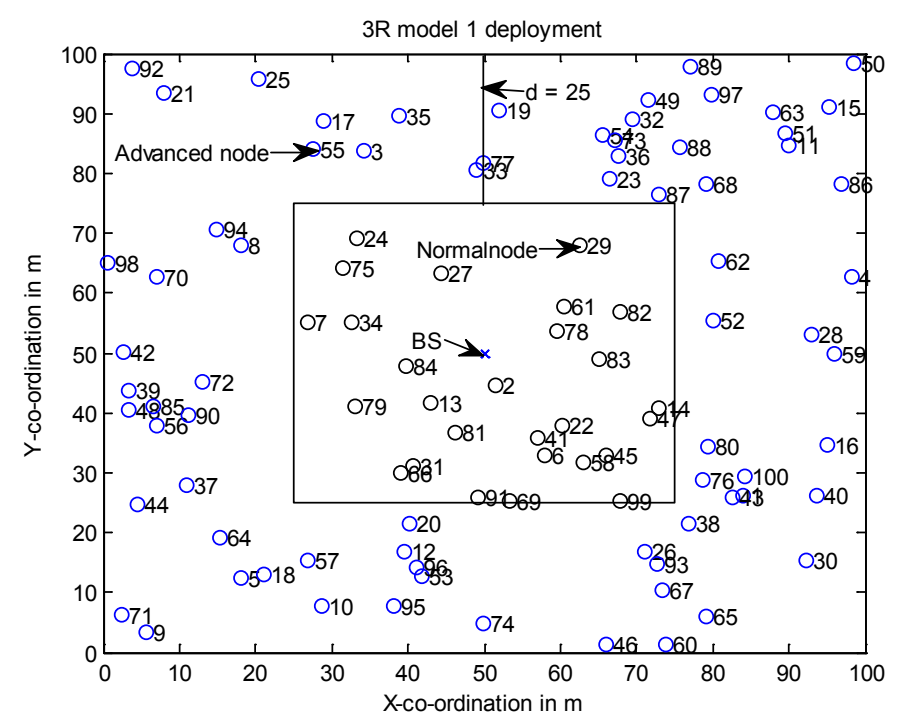

Figure 1. Node deployment in TWO Rim level Model-1.

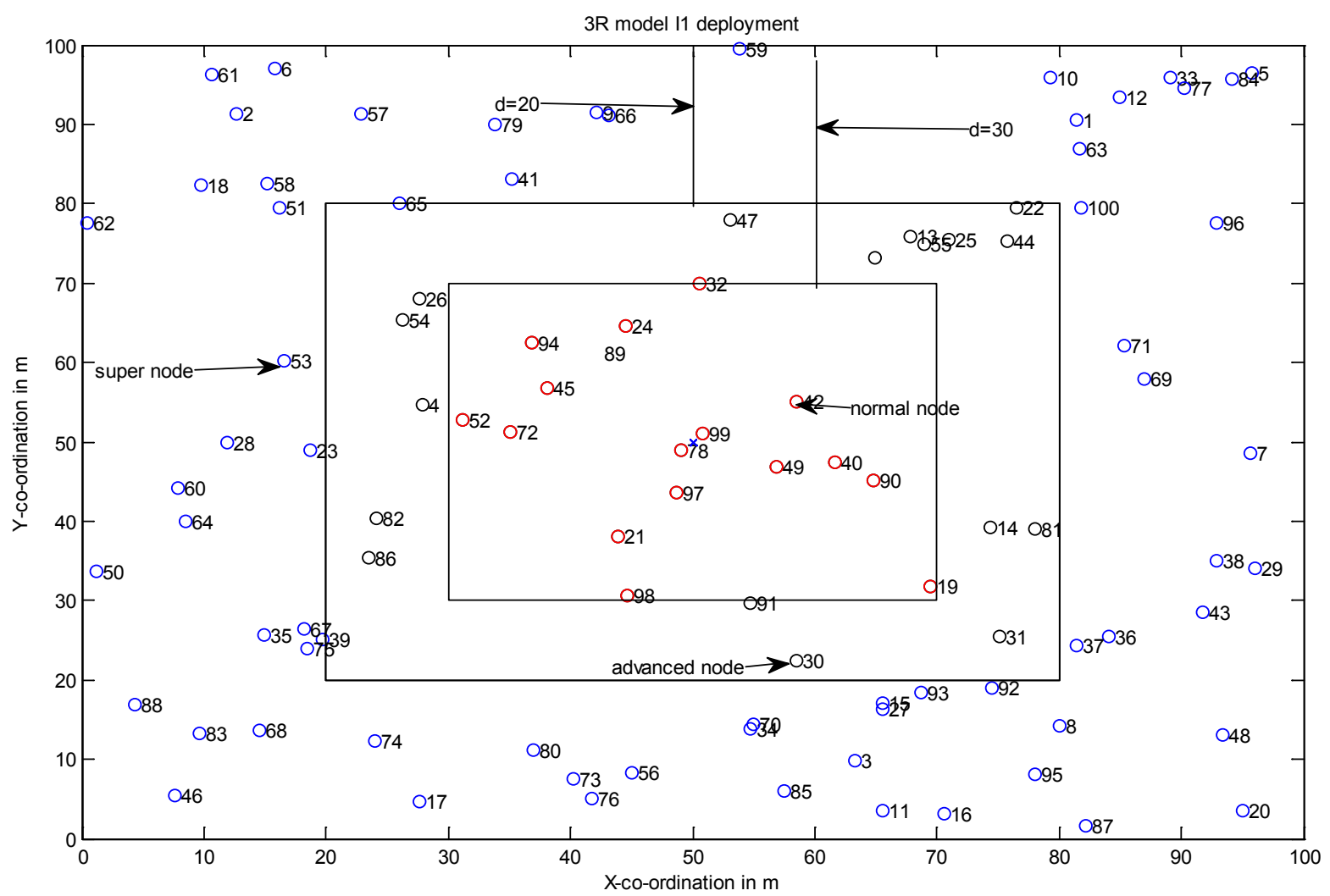

Figure 2. Node deployment in Three Rim level Model-2.

sume less energy for direct data transmission. The outer second rim has heterogeneous nodes Such as advanced node which is 1 Joule energy, these nodes also direct communication to the BS. Super nodes are deployed at outermost rim which have 1.5 Joule energy. The super nodes forming the clusters and follow the cluster head selection procedure. This new 3R method yield good performance of energy consumption and improve the network life time of the whole network. Consistently balanced the sensor nodes deployment in different energy level inside the rim and the operations are being formulated as the procedure of above said algorithm. 


\section{Simulation Results}

The establishments of all type of methods are executed as per the given algorithm and simulated consistently for SEP, ZSEP and 3R protocol model-1 and model-2. The comparison is done with new 3R technique under the same simulation conditions and values. Table 1 shows the comparison for all methods for the first dead node and last dead node in the number of simulation rounds as same SEP concept. As results shows that, the number of round in which first dead node is $779,781,880$ simulations or rounds with new protocol is increases to 1245 rounds. Same as last dead node extended up to 4020, when compare to the existing methods and 3R model-2 has $75 \%$ life time improvement than the SEP and other types of SEP concepts, which ultimately increases the lifetime of the whole network in terms of rounds. The optimum rim size also tested and it is given in Table 2.

Sensor nodes are randomly deployed at decided area, but inserted rim is fixed in size such as $5 \mathrm{~m}, 10 \mathrm{~m}, 15 \mathrm{~m}$ like that. If size of the rim is varied accordingly number of deployed node will be varied. Table 2 shows that the optimum rim size of network. From the table the rim size $25 \mathrm{~m}$ is optimum.

Figure 1 shows that the result between number of simulation rounds and number of dead nodes. From the Figure 1 clearly determined that the new 3R algorithm model-1 and model 2 yield the outperformance result and enhance the life time of networks than SEP, Such as 3R model-1 protocol gives 2890 rounds and 3R model-2 gives up to 4230 rounds of increased network lifetime as shown in Figure 3. The throughput and PDR results are given in Figure 4 and Figure 5 respectively. A reliable level of obtained good performance of 3R model 1 and 2 for throughput and PDR is evidenced in figures.

To increase the network lifetime, the method of deploying the sensor nodes is to be altered. They are deployed on the basis of rim. Rim size may be varied and heterogeneous node level may be varied, accordingly energy efficiency of the network ultimately increased.

Figure 4 shows that the result between number of rounds and number of packets delivery ratio of the sensor nodes. Packet delivery to the BS improved than the existing protocol SEP and ZSEP.

Number of packets send to clusters head and number of packets send to the base station within the given rounds and throughput also increased for the 3R model when compare to SEP and Z-SEP as shown in Figure 5.

Table 1. First and Last dead nodes in rounds for different models.

\begin{tabular}{llcccc}
\hline $\begin{array}{c}\text { Details of first/last dead } \\
\text { node for the different } \\
\text { methods }\end{array}$ & & \multicolumn{5}{c}{ Various Techniques } \\
\cline { 2 - 6 } & First dead node & 779 & ZSEP & 3R model-1 & 3R model-2 \\
\hline & Last dead node & 1089 & 1150 & 380 & 1245 \\
& & & & 3700 & 4020 \\
\hline
\end{tabular}

Table 2. Optimum Rim size with number of live nodes for rounds.

\begin{tabular}{|c|c|c|c|c|c|c|c|}
\hline \multirow{11}{*}{$\begin{array}{l}\text { Optimum RIM size for } \\
100 \text { node deployment. } \\
\text { Number of Rounds Vs } \\
\text { live nodes }\end{array}$} & \multicolumn{5}{|c|}{ Various Techniques } & \multirow[b]{2}{*}{$\mathrm{R}=25 \mathrm{~m}$} & \multirow[b]{2}{*}{$\mathrm{R}=30 \mathrm{~m}$} \\
\hline & Rounds & $\mathrm{R}=5 \mathrm{~m}$ & $\mathrm{R}=10 \mathrm{~m}$ & $\mathrm{R}=15 \mathrm{~m}$ & $\mathrm{R}=20 \mathrm{~m}$ & & \\
\hline & 500 & 03 & 00 & 00 & 00 & 00 & 00 \\
\hline & 700 & 03 & 00 & 01 & 01 & 00 & 00 \\
\hline & 1000 & 06 & 03 & 02 & 03 & 01 & 04 \\
\hline & 1500 & 48 & 22 & 11 & 05 & 03 & 06 \\
\hline & 2000 & 100 & 98 & 67 & 47 & 24 & 31 \\
\hline & 2500 & & & 68 & 56 & 33 & 47 \\
\hline & 3000 & & & 89 & 64 & 54 & 55 \\
\hline & 3400 & & & 100 & 96 & 81 & 75 \\
\hline & 3600 & & & & 100 & 90 & 100 \\
\hline
\end{tabular}




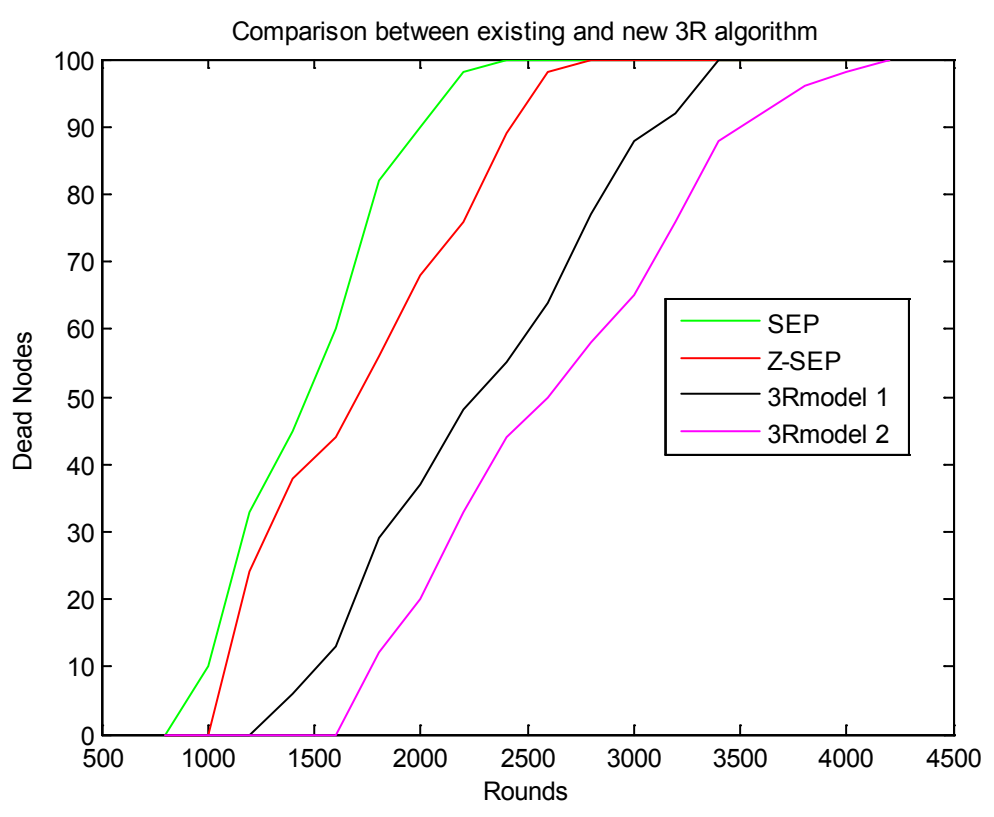

Figure 3. Result between number of rounds and number of dead nodes.

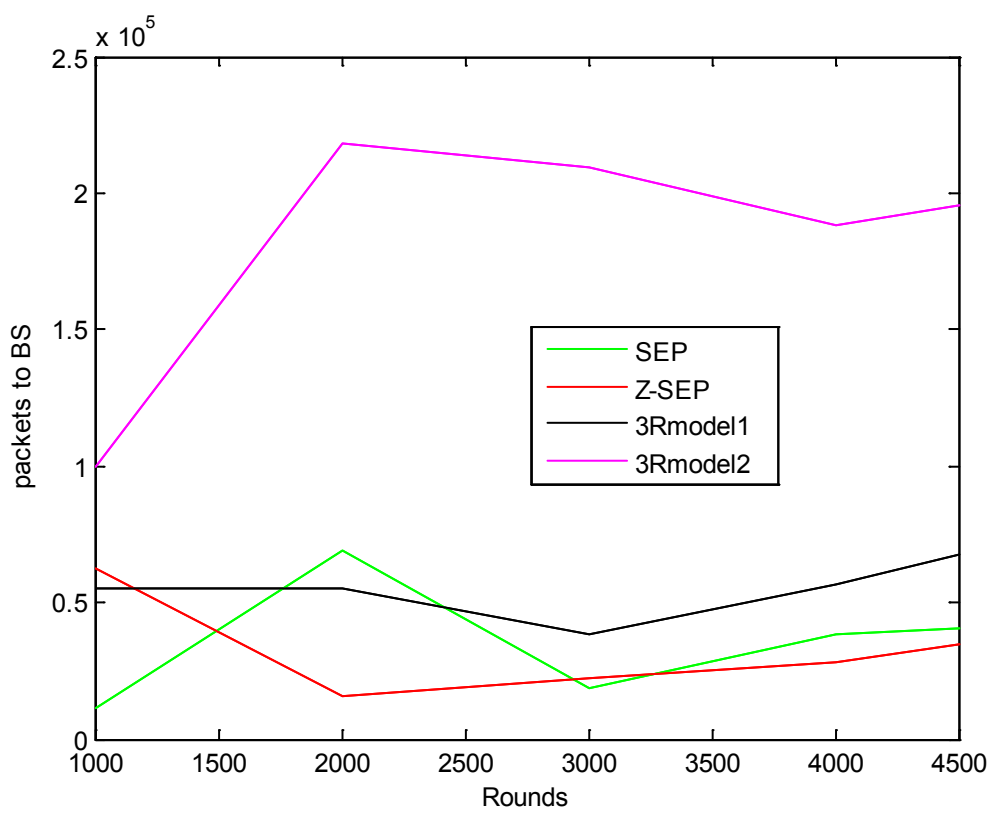

Figure 4. Result between numbers of rounds with PDR.

\section{Conclusion}

Deployment area, methods of node deployment and data communication strategies or techniques should help to save the energy in WSNs. By keeping the key point, the new $3 \mathrm{R}$ algorithm proves that changing the method of random sensor node deployment in different energy levels and the formation of cluster and cluster head selection cum communication of data transmission from the sensor node to cluster head and to base has increased in network life time when compared to SEP, Z-SEP. The performance varies when rim size changes. This 3R achieved its good performance by the procedure of the cluster formation and its burden of existing methods. In proposed 3R algorithm, Clusters are formed only in the extreme rim. Each $\mathrm{CH}$ in the extreme rim has to communicate to the BS, sensor nodes in other inner rim having very small distance to BS and it can communicate 


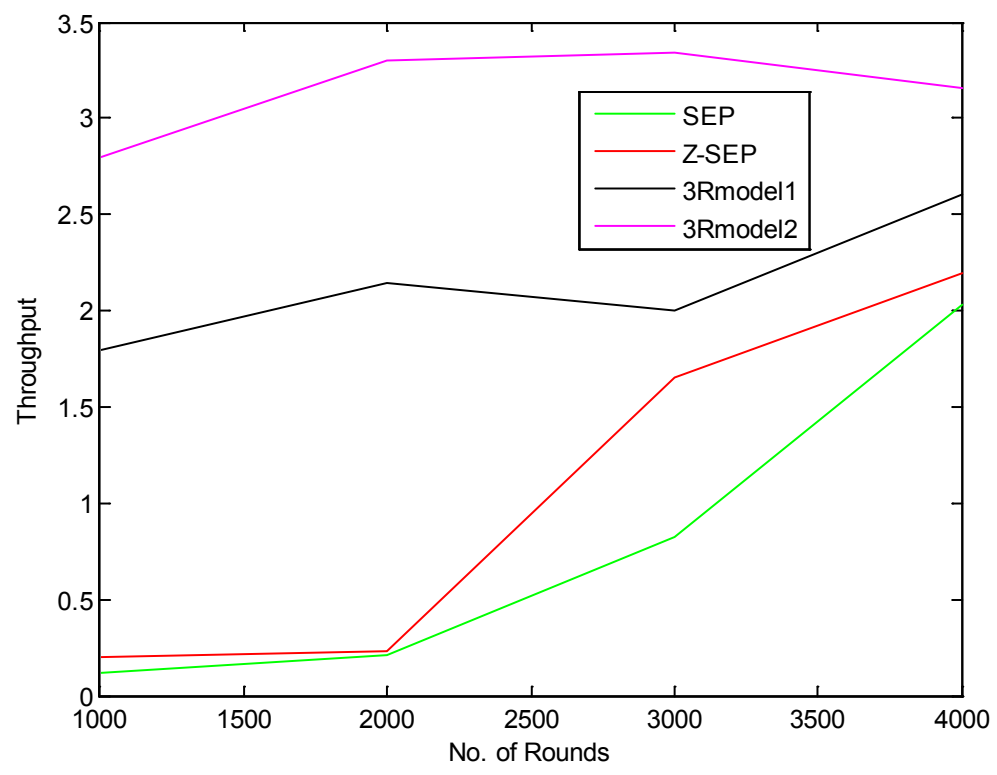

Figure 5. Result between numbers of rounds versus throughput of network for all methods.

the sensed data directly without forming clusters. In this way, energy efficiency is improved and achieved $70 \%$ than other types of SEP and better PDR with throughput for heterogeneous wireless sensor networks.

\section{References}

[1] Kumar, S. and Sah, P. (2015) A Survey on Energy Efficient Protocols for Wireless Sensor Network. International Journal of Computer Applications, 120, 4-8.

[2] Heinzelman, W., Chandrakasan, A. and Balakrishnan, H. (2000) Energy-Efficient Communication Protocol for Wireless Micro-Sensor Networks. Proceedings of the 33rd Hawaii International Conference on System Sciences, Hawaii, 4-7 January 2000, 1-10. http://dx.doi.org/10.1109/HICSS.2000.926982

[3] Heinzelman, W., Chandrakasan, A. and Balakrishnan, H. (2002) An Application-Specific Protocol Architecture for Wireless Micro-Sensor Networks. IEEE Transactions on Wireless Communication, 1, 660-670.

[4] Smaragdakis, I.M. and Bestavros, A. (2004) SEP: A Stable Election Protocol for Clustered Heterogeneous Wireless Sensor Networks. The Second International Workshop on Sensor and Actuator Network Protocols and Applications.

[5] Li, Q., Zhu, Q.X. and Wang, M.W. (2006) DEEC: Design of a Distributed Energy-Efficient Clustering Algorithm for Heterogeneous Wireless Sensor Networks. Computer Communications, 29, 2230-2237. http://dx.doi.org/10.1016/j.comcom.2006.02.017

[6] Faisal, S., Javaid, N., Javaid, A., Khan, M.A., Bouk, S.H. and Khan, Z.A. (2013) Z-SEP: Zonal Stable Election Protocol for Wireless Sensor Networks. Journal of Basic and Applied Scientific Research, 2, 1-9.

[7] Arya, G. and Chauhan, D.S. (2013) Modified Stable Election Protocol (M-SEP) for Hierarchical WSN. International Journal of Computer Applications, 79, 35-39. http://dx.doi.org/10.5120/13947-1926

[8] Liu, M., Cao, J., Chen, G. and Wang, X. (2009) An Energy-Aware Routing Protocol in Wireless Sensor Networks. Sensors, 9, 445-462. http://dx.doi.org/10.3390/s90100445

[9] Katiyar, N.C. and Soni, S. (2011) A Survey on Clustering Algorithms for Heterogeneous Wireless Sensor Networks. International Journal of Advanced Networking and Applications, 2, 745-754.

[10] Yi, S., Heo, J., Cho, Y. and Hong, J. (2007) PEACH: Power-Efficient and Adaptive Clustering Hierarchy Protocol for Wireless Sensor Networks. ELSEVIER Computer Communications, 30, 2842-2852.

[11] Wang, Y. (2008) Topology Control for Wireless Sensor Networks. In: Li, Y., Thai, M.T. and Wu, W., Eds., Wireless Sensor Networks and Applications, Signals and Communication Technology, Springer, US, 113-147. http://dx.doi.org/10.1007/978-0-387-49592-7_5

[12] Jeevitha, J. and Thirumaraiselvan, P. (2015) Multiparameter Based Energy Efficient Scheduling for Wireless Sensor Networks. International Journal of Innovative Research in Science, Engineering and Technology, 4, 550-557. 
[13] Jiwan, N., Patil, D.P. and Agarwal, G. (2013) Energy Consumption in Wireless Sensor Network. International Journal of Engineering Research \& Technology, 2, 1-4.

[14] Dietrich, I. and Dressler, F. (2009) On the Lifetime of Wireless Sensor Networks. ACM Transactions on Sensor Networks, 5, 1-39. http://dx.doi.org/10.1145/1464420.1464425

[15] Jebakumar, R. and Vivekanandhan, P. (2014) Distributed Data Storage through Secure Routing in WSNs. International Review on Computers and Software, 9, 478-486.

[16] Ari, A.A.A., Gueroui, A., Labraoui, N. and Yenke, B.O. (2015) Concepts and Evolution of Research in the Field of Wireless Sensor Networks. International Journal of Computer Networks \& Communications (IJCNC), 7, 81-98. http://dx.doi.org/10.5121/ijenc.2015.7106

[17] Bhattacharyya, B., Kim, T. and Pal, S. (2010) Comparative Study of Wireless Sensor Networks and Their Routing Protocols. Sensors, 10, 10506-10523. http://dx.doi.org/10.3390/s101210506

[18] Elleithy, A. and Liu, G. (2011) A Simulation Model for the Lifetime of Wireless Sensor Networks. International Journal of Ad Hoc, Sensor \& Ubiquitous Computing, 2, 1-15. http://dx.doi.org/10.5121/ijasuc.2011.2401

[19] Rathna, R. and Subarmanian, A.S. (2012) Improving Energy Efficiency in Wireless Sensor Networks. International Journal of Advanced Sensor Network Systems (IJASSN), 2, 21-27.

\section{Submit or recommend next manuscript to SCIRP and we will provide best service for you:}

Accepting pre-submission inquiries through Email, Facebook, Linkedin, Twitter, etc A wide selection of journals (inclusive of 9 subjects, more than 200 journals)

Providing a 24-hour high-quality service

User-friendly online submission system

Fair and swift peer-review system

Efficient typesetting and proofreading procedure

Display of the result of downloads and visits, as well as the number of cited articles

Maximum dissemination of your research work

Submit your manuscript at: http://papersubmission.scirp.org/ 